\title{
Therapeutische Hypothermie bei neonataler Asphyxie
}

\section{Therapeutic Hypothermia in Neonatal Asphyxia}

\section{Gortner}

Bibliografie DOI http://dx.doi.org/ 10.1055/s-0033-1363276 Klin Padiatr 2014; 226: 1-2 (c) Georg Thieme Verlag KG Stuttgart · New York ISSN 0300-8630

Korrespondenzadresse Prof. Dr. Ludwig Gortner Klinik für Allgemeine Pädiatrie und Neonatologie Kliniken für Kinder- und Jugendmedizin Universitätsklinikum des Saarlandes

Gebäude 9 66421 Homburg/Saar ludwig.gortner@uks.eu
Die neonatale hypoxisch-ischämische Enzephalopathie (HIE) wird bei rund 1 bis 3 pro 1000 Früh- und Neugeborenen in den entwickelten Ländern beobachtet und ist mit einer erheblichen Mortalität und neuropädiatrischen Folgemorbidität [7] behaftet. Nachdem nunmehr seit der Dekade des Gehirns ("decade of the brain") fast eine halbe Dekade vergangen ist, sind die wissenschaftlichen Bemühungen aus präklinischen und klinischen Untersuchungen der vergangenen 15 Jahre erfolgreich gewesen. Es konnte bei reifen Neugeborenen sowie späten Frühgeborenen von 36 Wochen Gestationsalter nach stattgehabter neonataler schwerer Asphyxie die Kühlungstherapie als Standard erfolgreich eingeführt werden. In der Mehrzahl der Studien war der prospektiv gewählte Endpunkt das kombinierte Kriterium der Reduktion der Mortalität oder schwerer entwicklungsneurologischer Residuen im Alter von 18 bis 24 Monaten. In der Regel wurde die entwicklungsneurologische sowie kognitive Testung von in Studien einbezogenen Neugeborenen unter anderem mittels der Bayley-II-Untersuchung durchgeführt [1]. Die nunmehr in der vorliegenden Ausgabe der Klinischen Pädiatrie in komprimierter Form publizierte Mitteilung auf dem Boden der entsprechenden Leitlinie für die Kühlungstherapie fasst die Indikation, Techniken, Begleituntersuchungen sowie Nachsorge bei von einer neonatalen Asphyxie betroffenen späten Frühgeborenen sowie reifen Neugeborenen zusammen [2].

Im Kontext einer jüngst publizierten Metaanalyse [6] unter Zugrundelegung von 11 klinisch kontrollierten Studien mit rund 1500 Kindern nach mittelschwerer bis schwerer HIE nach Asphyxie resultiert die therapeutische Hypothermie in eine signifikante Verminderung der oben genannten Zielvariable mit einer Reduktion des Risikos um rund 25\% im Vergleich zu Kontrollen. Die Anzahl von mit Hypothermie zu behandelnder Früh- und Neugeborener zur Reduktion des Merkmals Mortalität liegt bei 7, bezogen auf die entwicklungsneurologischen Handicaps die Anzahl zu behandelnder Kinder bei 8, um ein adverses Ereignis zu verhindern. Zu den langfristigen Konsequenzen einer schweren HIE zählen die infantile Zerebralparese, kognitive Entwicklungsstörungen und Anfallsleiden [7].

In den in die Metaanalyse einbezogenen Studien wurden durchgängig als unerwünschte Wirkungen der Therapie eine häufigere Sinusbradykardie und Thrombopenie in den Kühlungsgruppen beobachtet, in seltenen Fällen wurden Hautläsionen, insbesondere subkutane Fettgewebsnekrosen an den abhängigen Partien, d.h. den der Hy- pothermie direkt ausgesetzten Körperpartien, beschrieben. Langfristige unerwünschte Wirkungen der Hypothermietherapie wurden im Rahmen der genannten Nachsorgestudien nicht beobachtet.

Grundlagen für die Anwendung der therapeutischen Hypothermie nach experimenteller perinataler Asphyxie wurden bereits in größerer Zahl in den 90er-Jahren des vergangenen Jahrhunderts durchgeführt und waren die Basis für weitere Untersuchungen und den Beginn von klinisch-kontrollierten Studien in der vergangenen Dekade. Diese Untersuchungen wurden mit Nachdruck forciert, da zuvor durchgeführte klinisch-kontrollierte Studien mit dem Einsatz von Barbituraten, Allopurinol sowie Magnesiumsulfat keinen Nutzen belegen konnten [4]. Darüber hinaus gab es kleinere Studien, die mit Kalziumkanalblockern, Dexamethason und Naloxon als Opiatantagonist ebenfalls keinen Effekt der Therapie zeigen konnten bzw. wegen schwerer adverser Reaktionen abgebrochen wurden [11]. Tierexperimentell wurde die Pathophysiologie der HIE mit initialer Hypoxie bzw. Ischämie und nachfolgender Exzitotoxizität mit inflammatorischer Reaktion und einer Apoptose-/Nekrosesequenz in verschiedenen Modellen beschrieben und diente zur Entwicklung von therapeutischen Strategien, die neben der Hypothermie auch die Evaluation neuer additiver Konzepte beinhaltete [7,9]. Tierexperimentelle Modelle dienten weiter als Grundlage für die Entwicklung additiver Therapien zur therapeutischen Hypothermie. Diese beinhalten unter anderem Erythropoeitin, neuere Antikonvulsiva, z.B. vom Topiramat-Typ, bzw. Antagonisten der NMDA-Rezeptoren und regenerativer Therapieverfahren, unter anderem mittels der Applikation von Stammzellen $[3,5]$.

Daneben sind diese Untersuchungen Grundlage für die Planung weiterer klinisch-kontrollierter Studien, die unter anderem die Modifikation der Hypothermie dahingehend beinhalten, zum einen einen früheren Beginn und eine längere Dauer der Hypothermie (gegenwärtig 72 Stunden), zum anderen eine tiefere Hypothermie (gegenwärtig $33-34^{\circ} \mathrm{C}$ ) zum Gegenstand haben. Somit sind 2 große Linien der anlaufenden bzw. in Planung befindlichen, klinisch-kontrollierten Studien zu erkennen, die zum einen eine Optimierung der Behandlung mittels Veränderung der Hypothermiemodalitäten im obigen Sinne zum Gegenstand haben, zum anderen bei etabliertem, oben skizziertem Therapiestandard die Erprobung additiver Therapieformen. Diesen wissenschaftlichen Anstrengungen liegt das Ziel zugrunde, die Rate von $25 \%$ des Überlebens 
ohne schwere entwicklungsneurologische Residuen weiter zu verbessern.

Neben den genannten Ergänzungs- und Optimierungsstudien in den unterschiedlichen Ländern ist der Einsatz der therapeutischen Hypothermie in Schwellenländern ein wichtiger weiterer Studienansatz. Eine Metaanalyse auf dem Boden von 7 Studien mit insgesamt knapp 600 Kindern, bei denen die Hypothermie entweder mittels mit kaltem Wasser gefüllten Flaschen, gekühlten Gelpackungen sowie Kühlungskappen mit Wasserdurchfluss induziert wurde, war jüngst publiziert worden [8].

Diese zeigt, dass mit den gegebenen heterogenen Methoden bislang keine signifikante Verbesserung der neonatalen Mortalität resultiert [10]. Nachsorgeuntersuchungen über längere Zeiträume liegen hierbei nicht in systematischer Form vor.

Daher ist bei globaler Betrachtung neben der Verfeinerung der Therapie, die naturgemäß einen hohen Studienaufwand in den entwickelten Ländern bedeutet, die Entwicklung sicherer und effizienter Systeme in Schwellenländern zum Einsatz der therapeutischen Hypothermie bei neonataler HIE die vordringlichste Aufgabe.

Interessenkonflikt: Der Autor erklärt hiermit, dass kein Interessenkonflikt besteht.

\section{Literatur}

1 Chaudhary T, Walch E, Herold B et al. Predictive and concurrent validity of standardized neurodevelopmental examinations by the Griffiths scales and Bayley scales of infant development II. Klin Padiatr 2013; 225: 8-12

2 Flemmer A, Maier R, Hummler H. Treatment of neonatal asphyxia with a special focus on therapeutic hypothermia. Klin Padiatr 2014; 226: 29-37

3 Gortner L, Felderhoff-Muser U, Monz D et al. Regenerative therapy in neonatology: clincial perspectives. Klin Padiatr 2012; 224: 233-240

4 Gunn AJ. Cerebral hypothermia for prevention of brain injury following perinatal asphyxia. Curr Opin Pediatr 2000; 12: 111-115

5 Haase $R$, Korholz D, Herting $E$ et al. Rationale for regenerative treatment in neonatology. Klin Padiatr 2012; 224: 230-232

6 Jacobs SE, Berg M, Hunt $R$ et al. Cooling for newborns with hypoxic ischaemic encephalopathy. Cochrane Database of Systematic Reviews 2013; 1: CD003311

7 McLean C, Ferriero D. Mechanisms of hypoxic-ischemic injury in the term infant. Semin Perinatol 2004; 28: 425-432

8 Pauliah SS, Shankaran S, Wade A et al. Therapeutic hypothermia for neonatal encephalopathy in low- and middle-income countries: a systematic review and meta-analysis. PLoS ONE 2013; 8: e58834

9 Shankaran S. Hypoxic-ischemic encephalopathy and novel strategies for neuroprotection. Clin Perinatol 2012; 39: 919-929

10 Stichtenoth G, Demmert M, Bohnhorst B et al. Major contributors to hospital mortality in very-low-birth-weight infants: data of the birth year 2010 cohort of the German Neonatal Network. Klin Padiatr 2012; 224: $276-281$

11 Whitelaw A. Systematic review of therapy after hypoxic-ischaemic brain injury in the perinatal period. Semin Neonatol 2000; 5: 33-40 